\title{
La preservación de un idioma: ¿a partir de una política o una planificación linguística?
}

\author{
Milton Ascencio ${ }^{1}$ \\ Recibido en Noviembre 2012, aprobado en Abril 2013
}

\begin{abstract}
Resumen
En este artículo se presenta una revisión bibliográfica relacionada con la diversidad lingüística. Después de presentar argumentos que rechazan y defienden la preservación de lenguas amenazadas, se exponen los casos del hebreo y del irlandés como ejemplos del éxito y fracaso de políticas lingüísticas. Al final se plantea la planificación lingüística, como alternativa a la política lingüística, para que los hablantes aseguren la continuidad de su idioma.
\end{abstract}

Palabras claves:

Diversidad lingüística, lenguas amenazadas, política lingüística, planificación lingüística

\begin{abstract}
This article presents a literature review related to linguistic diversity. After presenting arguments for and against language maintenance, this article presents the cases of Hebrew and Irish, which represent the success and failure of language policies. At the end, language planning is presented as an alternative to language policy for those speakers who want to ensure the continuity of their traditional language.
\end{abstract}

Keywords:

Linguistic diversity, endangered languages, language policy, language planning

\section{Introducción}

El pasar del tiempo ha visto la aparición y desaparición de muchos idiomas. Por ejemplo, las lenguas romances que se hablan hoy en día, como el español, portugués y francés, son descendientes del latín y se consideran idiomas desde hace un poco más de mil doscientos años atrás. También hay idiomas que ya

1 Licenciatura en Idiomas de la Universidad de El Salvador. Maestría en Lingüística de la Universidad Estatal de Arizona. Director de la Escuela de Idiomas en la Universidad Don Bosco. E-mail: milton. ascencio@udb.edu.sv 
no se trasmiten en forma natural de generación en generación, como el latín mismo, y que no tienen funcionalidad dentro de una comunidad de hablantes por lo que se consideran lenguas muertas. Otros idiomas, como muchas lenguas indígenas en América, están en un peligro de extinción y en muchos casos podrían ya estar condenados a la desaparición.

Las razones por las que una lengua se ve en peligro de extinción son varias, pero normalmente son condiciones externas a la comunidad de hablantes que terminan por socavar la vitalidad del idioma. En Ascencio (2009) se presentan dos vías por las que una lengua puede llegar a la extinción. Una es por la vía rápida, en la que los hablantes son asesinados con impunidad, como fue el caso de los hablantes del náhuat asesinados por el General Martínez en 1932 en El Salvador. Una segunda vía es porque los mismos hablantes así lo deciden como resultado de un proceso acumulativo de desprestigio del idioma, en muchas veces en forma sistemática y deliberada, con la consecuente pérdida y abandono de su identidad cultural y de su lengua, su máxima expresión.

El camino a la muerte lingüística se caracteriza por un proceso gradual de reducción de hablantes. Wurm ${ }^{2}$ describe una lengua en peligro potencial cuando los niños dan preferencia a otro idioma y empiezan a olvidar su idioma materno. Si los únicos hablantes del idioma materno son adultos jóvenes, con muy pocos niños hablantes de esa lengua, el idioma ya está amenazado. Si los únicos hablantes son adultos, entonces el idioma está seriamente amenazado. Cuando los únicos hablantes del idioma son ancianos, el idioma está en una fase que se describe como "un idioma moribundo". Una vez que no quedan hablantes del idioma, se dice que esa lengua ha muerto.

La resucitación de una lengua muerta o la reversión, la revitalización y la preservación de lenguas amenazadas son intervenciones diferentes pero que tienen un objetivo en común: asegurar la continuidad del idioma. La resucitación y la preservación representan los extremos de un continuo en el que el primero pretende traer a la vida una lengua extinta mientras que el segundo presupone una acción deliberada de los hablantes para asegurar el uso y transmisión de su idioma. La reversión y revitalización se enfocan en mejorar la transmisión generacional del idioma e inyectar nuevos vigores al aumentar las áreas en las que la lengua puede ser usada tales como la escuela y el trabajo ${ }^{3}$.

\footnotetext{
2 E. Wurm., Strategies for language maintenance and revival. Language endangerment and language maintenance: introduction, p. 14.

3 Cf. N. Hornberg., y K., King, Language Revitalization in the Andes: can the schools reverse language shift? Journal of Multilingual and Multicultural Development, p. 428.
} 


\section{1. ¿Por qué preservar una lengua amenazada?}

Los argumentos a favor o en contra de la preservación de las lenguas amenazadas son subjetivos y podrían estar cargados de contenido ideológico. Ya sea a favor o en contra, la posición puede ser defendida o rechazada sin que esto demuestre el lado objetivo del asunto. A continuación se presentan tres argumentos en contra y en favor de la diversidad lingüística.

Un primer argumento en contra de la preservación de la diversidad lingüística es que en el mundo actual sólo los idiomas dominantes tienen la posibilidad de sobrevivir. En verdad, la configuración actual de las sociedades ha permitido que el idioma de los grupos sociales dominantes se imponga, volviéndose el idioma de las oportunidades para mejorar el empleo y la vida. El que los hijos hablen o no hablen un idioma dominante, como el inglés para el presente contexto, puede significar que en un futuro ellos estarán en ventaja o en desventaja frente a otros en la competencia por mejorar su condición social y de vida. Entonces, ¿para qué aferrarse a una lengua moribunda que no servirá para "dar de comer"?

Esta configuración actual de la sociedad recuerda a "la ley de la selva" en la que los más fuertes sobreviven al subyugar al más débil por la fuerza, en el que los muchos se someten a la voluntad de los pocos. Si bien es cierto que el hablar un idioma dominante da oportunidades de mejorar socialmente, también es cierto que ello se presta a prácticas de discriminación social. En acuerdo con Fishman ${ }^{4}$, una posición en favor de la diversidad lingüística es que los grupos minoritarios y sus idiomas deberían ser respetados y valorados por lo que son y por lo que encierran. Se debe convencer a quienes aún hablan una lengua amenazada que ellos tienen el derecho no sólo de mantener sus valores, creencias, identidad cultural; sino también de promoverlos y perpetuarlos a través de su idioma.

Además de los valores, creencias e identidad cultural, la diversidad lingüística permite acceder al conocimiento que se genera dentro de cada grupo social. Para algunos antropólogos como Saville-Troike ${ }^{5}$ cuando las lenguas desaparecen, todo el conocimiento relacionado con su cultura también desaparece. El idioma es un medio para comprender la organización social, las relaciones sociales y patrones de comportamiento que se transmiten de generación en generación a lo largo del proceso de socialización y culturización. En la misma línea, Bradley y Bradley señalan que los especialistas en etnobotánica están conscientes de

4 J. Fishman., Reversing language shift. Clevedon: Multilingual Matters, p. 32.

5 Cf. M. Saville-Troike., The ethnography of communication: an introduction, pp. 3 y 7. 
la pérdida de una lengua6. Debido a que cada grupo social se desarrolla en un sistema ecológico muy particular, éstos adquieren un conocimiento único sobre plantas y vida silvestre. Mucho de ese conocimiento que podría utilizarse para el desarrollo de medicinas curativas deja de transmitirse a lo largo de las generaciones cuando su lengua ancestral desaparece.

Un segundo argumento en contra de la preservación de la diversidad lingüística es que una vez que una lengua ha iniciado su camino hacia la extinción, no hay nada que hacer porque no hay suficientes recursos o porque ya es muy tarde para hacer algo.

La postura de que no hay nada que hacer por una lengua amenazada representa el pesimismo con respecto a los alcances que los esfuerzos puedan tener. Ese pesimismo fue identificado por un grupo ad hoc de expertos en lenguas amenazadas de la UNESCO${ }^{7}$ como una de las principales amenazas para las lenguas minoritarias. En el artículo se señala que muchos pueblos indígenas asocian su cultura con su condición de desventaja social, lo cual les hace pensar que no vale la pena mantener su idioma. Ante el pesimismo, Fishman ${ }^{8}$ recuerda que los defensores de la diversidad lingüística deben identificar las funciones y contextos que han de ser priorizados así como planificar los pasos a seguir para recuperar el uso de la lengua entre la comunidad de hablantes, en el corto y largo plazo. Los logros alcanzados harán que el pesimismo y el escepticismo se reduzcan.

Un tercer argumento en contra de la diversidad lingüística es que la pérdida de una lengua no implica la pérdida de la identidad cultural. Por lo tanto, preservar una lengua amenazada no es necesario.

Es cierto, la pérdida de la lengua materna no es necesariamente la pérdida completa de la identidad cultural. Ya se ha dicho antes que aunque una persona cambie su idioma materno por otro idioma, siempre mantiene su identidad cultural a través del folklore, la comida típica, las celebraciones, etc. Por ejemplo, varios estudios señalan que las personas de origen hispano en los Estados Unidos reemplazan el español por el inglés, aunque siguen sintiendo ser parte de e identificándose con la cultura hispana?.

6 Cf. D. Bradley, y M. Bradley (eds.), Language endangerment and language maintenance: introduction, p. xii.

7 UNESCO., Language vitality and endangerment. Report of the ad hoc expert group on endangered languages, p. 2.

8 J. Fishman., op. cit. p. 12.

9 Cf. D. Bills., Spanish home language use and English proficiency as differential measures of language maintenance and shift; L. Hart-Gonzalez, y M. Feingold. Retention of Spanish in the home. Internatioinal Journal of the Sociology of Language; Y. R. Solé., Bilingualism: stable or transitional? The case of Spanish in the United States. International Journal of the Sociology of Language. 
No obstante, la identidad cultural formada bajo el nuevo idioma no es exactamente igual a la identidad formada bajo el idioma materno. El cambio a un idioma dominante involucra cambio hacia la cultura del grupo dominante. Weisman ${ }^{10}$ condujo un estudio acerca de la identidad bicultural y las actitudes hacia el idioma entre los profesores de origen hispano en el sur de California. Su estudio concluyó que los profesores que habían sido absorbidos por el inglés tenían expectativas, actitudes y prácticas de la cultura dominante. Por el contrario, aquellos profesores que mantenía el español como su idioma materno mostraron una conexión fuerte con sus raíces culturales, y con frecuencia expresaban desafío hacia la cultura dominante. Para Weisman, dado que una lengua es la mejor forma de expresar intereses, valores y puntos de vista de la propia cultura, ésta se vuelve el criterio más sobresaliente para demostrar pertenencia a un grupo y su identidad cultural.

Fishman ${ }^{11}$ señala que la mayoría de culturas que pierden su idioma muestran el principio del “juego del dominó". Cuando una de las piezas de la cultura cae, otras piezas se debilitan y caen también. El idioma es una de las piezas esenciales dentro de una cultura, por lo que cuando el idioma desaparece otros rasgos culturales le siguen. Saville-Troike ${ }^{12}$ describe que muchos amerindios, quienes han perdido su idioma ancestral, se lamentan por su transculturación al punto de decir "no podemos ser indígenas si no tenemos un idioma indígena". En términos similares, Fishman argumenta que para ser 'un mejor miembro de la cultura', 'más auténtico', 'más leal' y 'más comprometido' es necesario preservar el idioma materno.

\section{2. ¿Política o planificación linguiística?}

Trabajar en pro de la diversidad lingüística implica tomar acciones pertinentes en los momentos oportunos que permitan revertir el proceso de erosión lingüística o aminorar las condiciones que la causan. Las intervenciones en la forma y en las funciones de una lengua normalmente se definen bajo los términos de "política lingüística" y "planificación lingüística” para indicar las formas en las que se pretende influir en el uso del idioma de una comunidad de hablantes en forma deliberada.

Ager ${ }^{13}$ define a la política lingüística como una planificación oficial que realizan aquellos que ostentan una autoridad pública. Como toda política pública, es

10 E. Weisman., Bicultural identity and language attitudes. Urban Education, pp. 203-225.

11 J. Fishman., op. cit. pp. 17-28.

12 M. Saville-Troike., op cit. p. 198.

13 D. Ager., Motivation in language planning and language policy. Clevedon: Multilingual Matters, pp. 5-6. 
una acción de arriba hacia abajo en la que el estado ejerce su poder político para cambiar, regular o mantener el comportamiento de los hablantes hacia su idioma. Ager también define la planificación lingüística como una influencia no oficial ejecutada por los individuos y su comunidad. La planificación lingüística, a diferencia de la política lingüística, es una iniciativa de abajo hacia arriba que conlleva una política social (metas, objetivos y estrategias) de la misma comunidad de hablantes para revertir el proceso de erosión de su idioma.

La política y la planificación lingüística tienen dos campos de aplicación que se conocen como la planificación del estatus y la planificación del corpus lingüístico. La planificación del estatus lingüístico, por un lado, tiene como propósito incidir en la determinación de las funciones sociales de una lengua en la comunidad de hablantes. Ello implica no sólo el reconocimiento del estado sino también la asociación del idioma con un incremento de sus funciones sociales como medio de instrucción en las escuelas, en la religión, la literatura, el trabajo y los medios de comunicación ${ }^{14}$. La planificación del corpus lingüístico, por otro lado, se refiere a las acciones que tienen por objetivo cambiar el cuerpo lingüístico del idioma: crear nueva terminología o seleccionar un sistema de escritura y una variedad del idioma como el estándar con el objetivo de "mejorar" la lengua. El objetivo de la planificación del corpus es hacer que el idioma adquiera un nuevo estatus social, más prestigio y nuevas funciones sociales. No obstante, para Cooper ${ }^{15}$ las modificaciones en la forma y las funciones de la lengua pueden tener propósitos que van más allá de lo lingüístico. Por ejemplo, la promoción de un idioma ha sido parte de los movimientos nacionalistas que buscan independencia y autonomía política como ha sido el caso del vasco, el catalán, el frisón y el irlandés.

\section{La política linguiística: el hebreo y el irlandés}

En la literatura especializada se encuentran casos de intervención en un idioma en la forma de políticas adoptadas por autoridades oficiales. El hebreo y el irlandés son, sin lugar a dudas, los casos más ilustrativos de intervenciones estatales. No sólo son los casos que más han cautivado la atención en cuanto a los esfuerzos gubernamentales que han implicado sino también porque son los mejores ejemplos del éxito y el fracaso de dichos esfuerzos.

Aunque los esfuerzos a favor del hebreo y del irlandés comenzaron casi al mismo tiempo, sus resultados fueron muy diferentes. Mientras que el irlandés

14 Cf. R. L. Cooper., Language planning and social change; W. Fierman., Language planning and national development.

15 Cf. R. L. Cooper, op. cit. p. 34. 
continuó su tendencia en reducción de hablantes y la República de Irlanda observó un incremento en hablantes de inglés, el idioma hebreo resucitó y se revitalizó hasta el punto que hoy en día se ha convertido en la lengua dominante de Israel.

La lengua hebrea fue prácticamente traída de entre los muertos. En un periodo de cincuenta años, a través de un movimiento de resucitación que comenzó alrededor de 1890, el hebreo se convirtió en el idioma oficial de Israel. El hebreo se volvió en un idioma vigoroso que se transmite a los niños en forma natural, un idioma que se usa diariamente, estandarizado a través de diccionarios, gramáticas escritas así como a través del sistema escolar desde la parvularia hasta la educación superior. El hebreo es un idioma moderno que se puede usar para hablar de cualquier tema ${ }^{16}$. La resucitación y reavivamiento del hebreo es con frecuencia descrito como una política lingüística exitosa, que contrasta con los esfuerzos para revitalizar el irlandés.

El irlandés es el primer idioma nacional de un estado independiente, Irlanda, pero que a la vez es el idioma local que se habla sólo por una minoría de la población. A finales del siglo diecinueve comenzó un movimiento nacionalista para revitalizar el irlandés y revertir la tendencia alcista del inglés; así, devolver el estatus al idioma irlandés. Fennell ${ }^{17}$ describe que desde 1920 hasta 1970 hubo una serie de medidas de gran alcance que fueron implementadas para revitalizar el irlandés. Estas medidas incluían una declaración del irlandés como el primer idioma nacional. Además, el irlandés era requisito para ingresar a la escuela, universidades y acceder a servicios públicos, así como para obtener becas, ayuda financiera y reconocimiento para aquellas familias cuyo hijos hablaban el irlandés. Estas y otras medidas fueron seguidas por la inauguración de medios de comunicación en irlandés a tal punto que el irlandés se esparció en todas las esferas de la vida cotidiana.

No obstante, a pesar de todos esos esfuerzos, el número de hablantes de irlandés no obtuvo una tendencia al alza como se esperaba. Los datos presentados por Fishman ${ }^{18}$ indican que en el Gaeltacht, Irlanda occidental, el porcentaje de personas que hablaban irlandés en la cotidianeidad cayó del $86.5 \%$ al $77.4 \%$ para 1981. Ó Riagáin ${ }^{19}$ también muestra una tendencia decreciente. El censo de 1996, según Ó Riagáin, reportó que un 43\% de los residentes en Gaeltacht

16 B. Spolsky., Language policy, p. 192.

17 D. Fennell., Can a shrinking linguistic minority be saved? Lessons from the Irish Experience. Minority languages today, p. 32.

18 J. Fishman., op. cit. p.124.

19 P. Ó Riagáin., Irish language production and reproduction 1981-1996. Can threatened languages be saved, p. 202. 
hablaba irlandés comparado con el $60 \%$ reportado en 1973 . Ahlqvist ${ }^{20}$ y Paulston ${ }^{21}$ concuerdan en señalar que el futuro del irlandés es incierto, aun en el corto plazo. Es natural, entonces, preguntarse ¿por qué los esfuerzos para revitalizar el irlandés no fueron tan exitosos?

Aunque se pueden argumentar varios aspectos negativos para explicar el porqué el movimiento a favor del irlandés no obtuvo los resultados deseados, hay tres aspectos que se deben discutir: el papel que juega el estado, el sistema educativo y las actitudes de los hablantes.

Fennell ${ }^{22}$ argumenta que el movimiento para revitalizar el irlandés falló porque descansaba en el supuesto que el estado podía salvar el irlandés. Aunque los irlandeses aceptaron y aplaudieron las iniciativas estatales en favor de su idioma, tanto los individuos, las familias y las comunidades continuaron el proceso de cambio hacia el inglés, proceso que había venido erosionando el irlandés por cuatro siglos.

Hay una creencia que para revivir o revitalizar una lengua, el primer paso es buscar el reconocimiento oficial del estado. A partir de esto, se cree que el estatus legal de la lengua garantizará en gran manera las probabilidades de sobrevivir porque así el estado estará obligado a promover su uso y obligado a aceptarlo como idioma oficial para todas las actividades sociales. Hay dos cosas que se deben señalar.

En primer lugar, es bastante arriesgado confiar sólo en las acciones de aquellos que ostentan el poder para llevar a cabo un tipo de acción a largo plazo -como el de revitalizar una lengua. Las prioridades e intereses de quienes están en un gobierno cambian con el tiempo. En este sentido Fishman ${ }^{23}$ señala que si por alguna razón el apoyo del estado se detiene, esto podría dejar un sentimiento de pesimismo y derrota, lo cual es peor que la misma amenaza que la ayuda prometía solventar. Sería más beneficioso hacer esfuerzos que se puedan mantener sin que su existencia dependa únicamente del apoyo externo.

En segundo lugar, una política gubernamental para mejorar el estatus de un idioma no garantiza un incremento del uso de ese idioma. El hebreo es un caso extraordinario ya que, como Cooper $^{24}$ señala, las condiciones en Palestina

20 A. Ahlqvist., Irish and Finland Swedish. Language endangerment and language maintenance: introduction, p. 40.

21 C. B. Paulston., Linguistic minoritites in multilingual settings, p. 90.

22 D. Fennell, op. cit. p. 37.

23 J. Fishman., (ed.) Can threatened languages be saved? Clevedon: Multilingual Matters, p. 13.

24 R. L. Cooper, op. cit. pp. 107-108. 
- la necesidad de una lengua franca, la familiaridad con el hebreo litúrgico y los incentivos materiales- favorecieron su rápido aprendizaje como lengua franca. En otras circunstancias, estas acciones gubernamentales no han tenido el mismo éxito. Dalby (2002:161-6) brinda un ejemplo de ello, señalando que las recientes políticas de los gobiernos de los Estados Unidos y Canadá para proteger las lenguas autóctonas no han logrado que las personas paren de cambiar su idioma por el inglés.

Aunque es cierto que en los últimos años ha habido un incremento en el apoyo para que los estados reconozcan los derechos de las personas para usar y mantener su idioma, así como para tener su propia religión, también es cierto que mantener el idioma propio es la responsabilidad de los hablantes como su derecho. Se espera que el estado respete este derecho lingüístico, así como que respete los derechos civiles; pero no se espera que un estado haga obligatorio el uso de ese idioma. La responsabilidad de preservar un idioma recae en los hablantes en primer lugar, antes que en el estado. El estado sólo, sin el deseo y sin la cooperación de los hablantes, no puede tener éxito en la restauración de la vitalidad de una lengua.

Otro punto que deriva del irlandés tiene que ver con el papel que el sistema educativo ha jugado en estos procesos. De acuerdo a Coady y Ó Laoire ${ }^{25}$ las políticas para revitalizar el irlandés tenían a las escuelas como pieza fundamental. El objetivo del gobierno de incluir el irlandés en el currículo nacional pasaba por crear una identidad del hablante de irlandés que gradualmente reemplazara al inglés hasta llegar a ser el irlandés el único medio de instrucción. Dicha política fue considerada como lo efectivo para revitalizar el irlandés.

Sin embargo, la efectividad de estas políticas educacionales ha sido cuestionada por varios. Paulston ${ }^{26}$ comenta que aunque la política era enseñar el irlandés a todos los niños en edad escolar, el inglés continuó en uso popular. Una razón para esta situación, comenta Coady y Ó Laoire ${ }^{27}$, es que el uso del irlandés fuera de la escuela no era reforzado ni se animaba para ello. Además, Flynn ${ }^{28}$ señala que a los estudiantes se les ha enseñado un poco de irlandés como una reflexión de su posición simbólica en el estado, mientras una minoría estudia el irlandés con el objetivo real de adquirirlo como otra lengua.

25 M. Coady, y M. Ó Laoire. Mismatches in language policy and practice in education: the case of gaelscoileanna in the Republic of Ireland. Language Policy, p. 143-146.

26 C. B. Paulston, op. cit. p. 81.

27 M. Coady, y M. Ó Laoire., op. cit. p. 149.

28 D. Flynn., Irish in the school curriculum: a matter of politics, p. 75. 
Hay dos condiciones para que exista una contribución efectiva de la escuela en la preservación de una lengua amenazada: la adquisición de esa lengua antes de la escuela y el uso después de la escuela. Primero, la adquisición de una lengua amenazada se puede dar a través de programas de inmersión. Brandt y Ayoungman ${ }^{29}$ mencionan que los programas de inmersión han dado resultados positivos en varias lenguas como makah en Washington, cree en Canadá y el mohawk en los Estados Unidos y Canadá. Probablemente, el programa de inmersión más exitoso se ejemplifica a través de los “language nests" o nidos lingüísticos para la adquisición y desarrollo de una lengua en Nueva Zelanda entre los grupos hablantes de maorí y hawaiano. Los maoríes y hawaianos implementaron sus propios programas de inmersión pre-escolares, comenzando en 1984. Estos se extendieron a la primaria y luego a la secundaria. Francis y Reyhner ${ }^{30}$ comentan que como no se podían emplear hablantes natales del idioma entre la generación de padres, fueron los abuelos los que se comprometieron e hicieron la mayor parte de la enseñanza. Continúan diciendo que el punto crucial para revitalizar el maorí y el hawaiano no fueron los programas de la escuela primaria sino los programas de inmersión pre-escolar.

Segundo, el refuerzo del idioma amenazado fuera de la escuela involucra la motivación de los padres para aprender y para usar su idioma de manera que las generaciones posteriores aprendan ese idioma, antes de ir a la escuela. El refuerzo fuera de la escuela también debe ser orientado a los adolescentes y a los adultos jóvenes de manera que se asegura la continuidad del idioma. Ante esto, Fishman ${ }^{31}$ argumenta que si la lengua amenazada no se asegura antes de ir a la escuela y que si no se da su socialización a nivel de familia, vecindario y comunidad, la continuidad de una lengua no puede ser garantizada.

La evidencia del estado y los esfuerzos de la escuela para preservar una lengua sugieren que los únicos que pueden salvar una lengua en peligro de extinción son los mismos hablantes de esa lengua. ¿Por qué es que algunos grupos se aferran a su idioma y cultura mientras que otros parecen estar listos para enterrar su cultura, identidad e idioma? Bradley ${ }^{32}$ dice que un factor crucial en la preservación de un idioma es la actitud de la comunidad de hablantes hacia su idioma.

29 E. A. Brandt, y V. Ayoungman. Language renewal and language maintenance: a practical guide, p. 47.

30 N. Francis, y J. Reyhner., Language and literacy teaching for indigenous education, pp. 32-39.

31 J. Fishman., Reversing language shift. Clevedon: Multilingual Matters, p. 374.

32 D. Bradley., Language attitudes. Language endangerment and language maintenance. Language endangerment and language maintenance: introduction, p. 1. 
Huss $^{33}$ observa que las actitudes positivas no necesariamente garantizan la continuidad de un idioma. Ya se ha mencionado en otros lugares que el camino hacia la muerte lingüística esta pavimentado con buenas intenciones. Paulston ${ }^{34}$ nota que el irlandés es altamente valorado por la mayoría de hablantes como un símbolo de su identidad; no obstante, eso no significa que el número de hablantes haya aumentado grandemente. En su trabajo sobre las opiniones, actitudes y valoraciones de los hablantes del navajo acerca de los programas de preservación de dicho idioma, Clark ${ }^{35}$ concluye que la mayoría de hablantes de navajo tienen una actitud positiva hacia esta lengua ancestral. Sin embargo, Spolsky ${ }^{36}$ reporta que para 1992 la mitad de los niños navajos en programas iniciales eran ya hablantes de inglés y que para 1993 menos de un tercio de los niños párvulos hablaban un navajo fluido. Esta tendencia ya ha sido observada entre los hablantes de origen hispano en los Estados unidos. Un alto porcentaje de ellos reflejan abiertamente una actitud positiva hacia el español, pero al mismo tiempo los estudios concuerdan que hay una clara tendencia de cambio hacia el inglés.

Fennell ${ }^{37}$ argumenta que un idioma erosionado no se puede salvar por la acción de un grupo de personas con buenos propósitos que no pertenecen a la comunidad en cuestión. Esta observación tiene implicaciones para un programa de revitalización. Primero, para que un idioma sea revitalizado, éste debe contar con la participación de los miembros de la comunidad de hablantes comprometidos en revertir la erosión lingüística de su idioma. Segundo, para restaurar el idioma, los esfuerzos han de orientarse y enfocarse en cambiar el comportamiento lingüístico de los hablantes más que sus actitudes hacia su idioma. Brandt y Ayoungman ${ }^{38}$ concuerdan que hay que cambiar el comportamiento antes que las actitudes ya que las actitudes con frecuencia cambian como resultado de los cambios en el comportamiento, y no al revés. Enfocarse en cambiar las actitudes podría retrasar un proyecto en gran manera.

Los argumentos revisados hasta el momento sugieren que una política por si misma o la escuela desarticulada de otros dominios que fortalecen la transmisión generacional no pueden tener el éxito deseado para revitalizar una lengua amenazada. Las intervenciones para recuperar la vitalidad de una lengua deben y necesitan involucrar y comprometer a los hablantes de esa lengua, quienes en

33 L. Huss., Reversing language shift in the far North, p. 29.

34 C. B. Paulston, op. cit. p. 86.

35 D. C. Clark., Language maintenance in the Navajo nation: native insights, opinions, and attitudes.

36 B. Spolsky., op. cit. p. 203-204.

37 D. Fennell, op. cit. p. 39.

38 E. A. Brandt, y V. Ayoungman, op. cit. p. 58. 
última instancia son los responsables por asegurar la transmisión generacional de su idioma. Un punto similar es adoptado bajo el enfoque de la planificación lingüística, lo cual se aborda a continuación.

\section{La planificación linguiística}

La planificación lingüística es un tipo de micro-planificación llevada a cabo por individuos y grupos interesados en revertir el proceso de erosión lingüística en el que su idioma está inmerso. En acuerdo con Fishman ${ }^{39}$, la planificación lingüística es una alternativa para aquellos grupos minoritarios que quieren restaurar y preservar su lengua ya que les da la oportunidad de regular sus propias vidas y a adaptar las políticas a sus necesidades más que ser "el objeto" de esas políticas que vienen desde "afuera" y desde "arriba".

Para Cooper ${ }^{40}$ la restauración y la preservación de un idioma necesitan de un movimiento, como los movimientos sociales que buscan reformar o revolucionar una sociedad y cuyas acciones determinan las políticas en un esfuerzo deliberado por ejercer un cambio social. A diferencia de la política lingüística, el resultado que se obtiene de un movimiento lingüístico se fundamenta en la misma comunidad lingüística. En caso de no existir ese movimiento lingüístico, se debe construir a través de un proceso de clarificación ideológica. Huss ${ }^{41}$ define clarificación ideológica como un proceso para despertar conciencia y para darse cuenta que se necesita el esfuerzo de cada uno de los hablantes para que la revitalización tenga éxito. El preservar un idioma requiere de individuos y hablantes comprometidos quienes desarrollan acciones apropiadas a favor de su idioma, aún en la ausencia de la ayuda estatal.

Fishman ${ }^{42}$ hace una exposición detallada de la planificación lingüística. En ella, se propone una escala a la cual Fishman llama "Graded Intergenerational Disruption Scale (GIDS)". La utilidad de esta escala es que propone intervenciones, estrategias y acciones, para revitalizar la lengua a partir del grado de ruptura en la transmisión entre generaciones de padres a hijos. A diferencia de otras escalas para medir la vitalidad de una lengua, como en Krauss 1992, UNESCO 2003, Wurm 2000, 2001, la escala propuesta por Fishman orienta y prioriza acciones a partir del grado de deterioro de la lengua. Una vez identificado el estado de la lengua, se procede a reparar y solucionar las causales para luego moverse hacia arriba en la escala.

39 En L. Huss., op. cit. p. 38.

40 R. L. Cooper, op. cit. p. 167.

41 L. Huss., op. cit. p. 29.

42 J. A. Fishman., Joshua A. Reversing language shift. Clevedon: Multilingual Matters, pp. 88-109. 


\begin{tabular}{|l|l|}
\hline El ámbito "fuerte" & $\begin{array}{l}\text { 1. Integración de la lengua a la educación superior, el } \\
\text { gobierno y los medios de comunicación. } \\
\text { 2. Integración de la lengua a los servicios públicos } \\
\text { 3. Integración de la lengua a las áreas de trabajo y } \\
\text { ocupaciones. } \\
\text { 4. Integración de la lengua a la educación formal }\end{array}$ \\
\hline \multirow{2}{*}{ El ámbito "débil" } & $\begin{array}{l}\text { 5. Integración de la escuela para enseñar a leer y } \\
\text { escribir en la lengua a los adultos, ancianos y niños. } \\
\text { 6. Promoción del uso de la lengua dentro de la familia, } \\
\text { el vecindario y la comunidad. } \\
\text { 7. Movilización de ancianos que aún hablan la lengua } \\
\text { para que la enseñen a las generaciones jóvenes. } \\
\text { 8. Reconstrucción de la lengua amenazada. }\end{array}$ \\
\hline
\end{tabular}

La escala anterior se explica en dos bloques. Del 8 al 5 en la escala, el ámbito débil, tiene como propósito asegurar la transmisión y la preservación de la lengua amenazada entre generaciones, tomando en cuenta las funciones vernáculas como punto de partida para luego continuar hacia arriba. Este ámbito débil supone que tanto el idioma dominante como el idioma minoritario pueden llegar a coexistir, estableciendo funciones y contextos propios para su uso. Este ámbito débil es el ámbito del sufrimiento y sacrificio, pues conlleva el arduo trabajo de convencer y despertar conciencia en la comunidad de hablantes que tienen la oportunidad y responsabilidad de asegurar la continuidad de su lengua.

Del 4 al 1 en la escala, el ámbito fuerte, tiene el objetivo de obtener poder y movilidad social. Si la comunidad lingüística consigue asegurar la continuidad de su idioma (pasos 8 al 5), es el momento de tomar acciones legales. Fishman ${ }^{43}$ sugiere que hacer provisiones legales antes de este ámbito sería un ejemplo de legis sine moribus vanae, "las leyes sin moral son vanas".

La aplicación de esta escala parte de un análisis del estado actual de una lengua. Por ejemplo, en el caso que un idioma no cuente con tradición escrita, es imperante registrarlo y reconstruirlo a través de un sistema de escritura, gramáticas y diccionarios. Si los únicos hablantes son adultos o ancianos, la implementación de programas de inmersión pre-escolar, como los “language nests", podrá ser un recurso de gran valor para integrar a los ancianos de la comunidad de hablantes en los esfuerzos de recuperación de su idioma. A la vez, se han de planificar las acciones encaminadas para la promoción del uso del idioma en los vecindarios o lugares frecuentados por los hablantes tales como señalizaciones, nombres, frases populares, etc. Los esfuerzos deben enfocarse en visibilizar la lengua y hacer que el idioma comience el camino hacia la 
normalización en su uso cotidiano. Estas acciones deben estar acompañadas por un trabajo incansable por enseñar el idioma, aunque como segunda lengua, a jóvenes y adultos.

Analizar el grado de deterioro y reflexionar-planificar acciones encaminadas a revertir el deterioro lingüístico es una tarea imperante e igualmente importante que los hablantes de una lengua amenazada han de tomar muy en serio, si quieren la continuidad de su idioma. El ámbito débil es el que al final determinará el éxito o el fracaso de un proceso de reversión o revitalización de un idioma. A diferencia de una política basada en la escuela y educación formal como instrumento de preservación de una lengua, en este enfoque los dominios de la familia-vecindario-comunidad son los que aseguran o no la continuidad de un idioma antes que los niños asistan a la escuela. Diferente a una política estatal que demanda aprender un idioma, este enfoque tiene como fundamento el compromiso de los hablantes en recuperar su lengua ancestral. Es el esfuerzo e involucramiento de los hablantes el motor que hace que un proceso de recuperación o revitalización tenga el éxito esperado. Superado este ámbito débil, habrá un mejor escenario y condiciones más fortalecidas para demandar reconocimiento del estado así como reclamar leyes que le den un estatus a la lengua amenazada y un lugar en la sociedad.

\section{Conclusión}

Realizar esfuerzos para preservar la diversidad lingüística va en la línea de defender los derechos inalienables que tienen todos para mantener y preservar su identidad cultural. Los grupos minoritarios, como muchos pueblos indígenas, tienen el derecho a existir como tales, promover y preservar sus culturas e idiomas. La importancia de preservar las lenguas amenazadas va más allá de mantener un simple medio de comunicación. Los idiomas encierran valores, experiencias, conocimiento y son la máxima expresión de la identidad cultural. ¿Cuántos lingüistas se necesitan para recuperar una lengua amenazada? Una respuesta muy bien podría ser: ninguno. Se necesita un movimiento, un grupo de personas de la comunidad de hablantes que se sientan identificados con su cultura y que tengan el deseo y el compromiso de planificar intervenciones que reviertan la erosión de su idioma a partir de acciones pertinentes. En la línea de la planificación lingüística, es preferible que la ejecución de estas acciones para garantizar la continuidad de un idioma venga desde el interior de la comunidad de hablantes, sin que estas acciones dependan necesariamente de políticas oficiales. La escala GIDS propuesta por Fishman es una oportunidad de reflexión y análisis sobre las acciones que se realicen a favor de una lengua en peligro de extinción. 


\section{Referencias}

Ager, Dennis. Motivation in language planning and language policy. Clevedon: Multilingual Matters. 2001.

Ahlqvist, Anders. Irish and Finland Swedish. Language endangerment and language maintenance: introduction, ed. by David Bradley and Maya Bradley, 40-58. New York: RoutledgeCurzon. 2001.

Ascencio, Milton. La pérdida de una lengua: el caso del náhuat. Teoría y Praxis 14. 65-78. 2009.

Azurmendi, Maria-Josi, Erramun Bachoc, y Francisca Zabaleta. Reversing language shift: the case of Basque. Can threatened languages be saved?, ed. by Joshua A. Fishman, 234-59. Clevedon: Multilingual Matters Ltd. 2001.

Bills, Gardland D., Alan Hudson, y Eduardo Hernández Chávez. Spanish home language use and English proficiency as differential measures of language maintenance and shift. Southwest Journal of Linguistics 19:1.11-27. 2000.

Bradley, David, y Maya Bradley (eds.) Language endangerment and language maintenance: introduction. New York: RoutledgeCurzon. 2001.

Bradley, David. Language attitudes. Language endangerment and language maintenance. Language endangerment and language maintenance: introduction, ed. by David Bradley and Maya Bradley, 1-9. New York: RoutledgeCurzon. 2001.

Brandt, Elizabeth A., y Vivian Ayoungman. Language renewal and language maintenance: a practical guide. Canadian Journal of Education 16:2.4277. 1989.

Clark, Diane Chardon. Language maintenance in the Navajo nation: native insights, opinions, and attitudes. Thesis, Arizona State University. 1995.

Coady, Maria, y Muiris Ó Laoire. Mismatches in language policy and practice in education: the case of gaelscoileanna in the Republic of Ireland. Language Policy 1.143-58. 2002. 
Cooper, Robert L. Language planning and social change. Cambridge: Cambridge University Press. 1989.

Curruchiche, Germán. Procesos de oficialización de los idiomas indígenas de Guatemala. La planificación lingüística en países multilingües de Abya Yala, ed. by Alfonso Buc Choc B’ahlam, 313-20. Guatemala: ALMG. 1995.

Dalby, Andrew. Language in danger. London: The Penguin Press. 2002.

Fennell, Desmond. Can a shrinking linguistic minority be saved? Lessons from the Irish Experience. Minority languages today, ed. by Einar Haugen, J. Derrick McClure, and Derick Thomson, 32-9. Edinburgh University Press. 1980.

Fierman, William. Language planning and national development. New York: Mouton de Gruyter. 1991.

Fishman, Joshua A. (ed.) Can threatened languages be saved? Clevedon: Multilingual Matters Ltd. 2001.

Fishman, Joshua A. Reversing language shift. Clevedon: Multilingual Matters. 1991.

Flynn, Donal. Irish in the school curriculum: a matter of politics. The Irish Review, 14. 74-80. 1993.

Fought, Carmen. Language attitudes. Chicano English, 193-225. New York: Palgrave. 2003.

Francis, Norbert, y Jon Reyhner. Language and literacy teaching for indigenous education. Clevedon: Multilingual Matters LTD. 2002.

Gorter, Durk. A Frisian update of reversing language shift. Can threatened languages be saved?, ed. by Joshua A. Fishman, 215-33. Clevedon: Multilingual Matters Ltd. 2001.

Grimes, Barbara F. Global language viability: causes, symptoms and cures for endangered languages. Notes on Linguistics 4:4.205-23. 2001. 
Hart-Gonzalez, Lucinda, y Marcia Feingold. Retention of Spanish in the home. Internatioinal Journal of the Sociology of Language 84.5-34. 1990.

Hornberg, Nancy H., y Kendall A. King. Language Revitalization in the Andes: can the schools reverse language shift? Journal of Multilingual and Multicultural Development 17:6.427-41. 1996.

Huss, Leena. Reversing language shift in the far North. UPPSALA: Acta Universitatis Upsaliensis. 1999.

Krauss, Michael. The world's languages in crisis. Language 68:1.4-10. 1992.

Maxwell, Judith. La unificación lingüística y la oficialización de las lenguas mayas de Guatemala. La planificación lingüística en países multilingües de Abya Yala, ed. by Alfonso Buc Choc B’ahlam, 279-99. Guatemala: ALMG. 1995.

Ó Riagáin, Pádraig. Irish language production and reproduction 1981-1996. Can threatened languages be saved?, ed. by Joshua A. Fishman, 195-214. Clevedon: Multilingual Matters Ltd. 2001.

Paulston, Christina Bratt. Linguistic minoritites in multilingual settings. Amsterdam: John Benjamin Publishing. 1994.

Saville-Troike, Muriel. The ethnography of communication: an introduction, third edition. Malden, MA: Blackwell Publishers Ltd. 2003.

Silva-Corvalán, Carmen. Conflicting loyalties. Language contact and change: Spanish in Los Angeles, 168-86. Oxford: Clarendon Press. 1994.

Solé, Yolanda Russinovich. Bilingualism: stable or transitional? The case of Spanish in the United States. International Journal of the Sociology of Language 84.35-80. 1990.

Spolsky, Bernard. Language policy. Cambridge: Cambridge University Press. 2004.

Strubell, Miquel. Catalan a decade later. Can threatened languages be saved?, ed. by Joshua A. Fishman, 260-83. Clevedon: Multilingual Matters Ltd. 2001. 
UNESCO. Language vitality and endangerment. Report of the ad hoc expert group on endangered languages.

Weisman, Evelyn Marino. 2001. Bicultural identity and language attitudes. Urban Education 36:2.203-25. 2003.

Wurm, Stephen. Language endangerment in the greater pacific area. Language death and language maintenance: theoretical, practical, and descriptive approaches, ed. by Mark Janse and Sijmen Tol, 15-47. Philadelphia: John Benjamins Publishing Company. 2000.

Wurm, Stephen. Strategies for language maintenance and revival. Language endangerment and language maintenance: introduction, ed. by David Bradley and Maya Bradley, 11-23. New York: RoutledgeCurzon. 2001. 